\title{
Amplification of distinct $\alpha$-synuclein fibril conformers through protein misfolding cyclic amplification
}

\author{
Byung Chul Jung ${ }^{1,2}$, Yoon-Ju Lim ${ }^{1}$, Eun-Jin Bae ${ }^{1}$, Jun Sung Lee ${ }^{1}$, Min Sun Choi ${ }^{1}$, Michael K Lee ${ }^{3}$, He-Jin Lee ${ }^{4}$, \\ Yoon Suk Kim ${ }^{2}$ and Seung-Jae Lee ${ }^{1}$
}

Amyloid fibril formation has been implicated in the pathogenesis of neurodegenerative diseases. Fibrillation generates numerous conformers. Presumably, the conformers may possess specific biological properties, thus providing a biochemical framework for strains of prions. However, the precise relationship between various fibril conformers and their pathogenic functions has not been determined because of limited accessibility to adequate amounts of fibrils from tissue samples. $\alpha$-Synuclein is one such protein, and it has been implicated in Parkinson disease. Using a technique known as protein misfolding cyclic amplification, originally developed for amplifying prions, we established a procedure through which the amplification of $\alpha$-synuclein fibrils is possible. With a trace amount of seeds, we succeeded in amplifying $\alpha$-synuclein fibrils. The replication of the seeds was faithful in terms of conformation even after multiple rounds of cyclic amplification. Moreover, two transgenic mouse strains each representing a distinct synucleinopathy were used to investigate different conformers by using this technique. The amplified $\alpha$-synuclein fibrils derived from the tissue extracts of these two strains led to the production of two different fibril conformers with distinct proteinase $\mathrm{K}$ digestion profiles. Together, our results demonstrated that a trace amount of $\alpha$-synuclein fibrils in tissue extracts could be amplified with their conformations conserved. This procedure should be useful in amplifying $\alpha$-synuclein fibrils from the brains and body fluids of patients afflicted with synucleinopathies and may serve as a potential diagnostic tool for Parkinson disease and other synucleinopathies.

Experimental \& Molecular Medicine (2017) 49, e314; doi:10.1038/emm.2017.1; published online 7 April 2017

\section{INTRODUCTION}

The etiology of most neurodegenerative diseases remains elusive but is now generally accepted to be associated with the aggregation of particular proteins. ${ }^{1}$ The neuropathology of these proteins is manifested in accordance with certain neurological diseases. For example, Alzheimer disease (AD) has two proteinaceous hallmarks: senile plaques produced by the aggregation of amyloid $\beta(\mathrm{A} \beta)$ and neurofibrillary tangles comprising hyperphosphorylated tau proteins. $^{2}$ The aggregation of another protein, $\alpha$-synuclein, encapsulates several representative disorders, including Parkinson disease (PD), multiple system atrophy (MSA) and dementia with Lewy Bodies (DLB), which are collectively referred to as synucleinopathies. ${ }^{3}$ The common denominator among these protein aggregates is the breakdown of the protein quality control system, thus resulting in the accumulation and voluntary assembly of misfolded proteins. ${ }^{4}$
The aggregates detected in pathological structures in disease-afflicted brains share structural similarities, reflecting a filamentous morphology and cross $\beta$-sheet secondary structure; these aggregates are classified as amyloid fibrils. ${ }^{5}$ The fibrils develop through a nucleation-dependent process in which a long lag phase is followed by an exponential growth phase. ${ }^{6}$ However, this classical process can be manipulated such that the addition of preformed fibrils expedites the growth from monomers to fibrils. ${ }^{6}$ This phenomenon, referred to as seeded fibrillation, is the molecular foundation that facilitates prion infectivity and aggregate spreading in neurodegenerative diseases. $^{7,8}$

Although amyloid fibrils bear structural resemblance to an extent, there are variations among these molecules. These slight differences create various species of fibril conformers. ${ }^{9}$ Therefore, strains in prion diseases and heterogeneous conformers in neurodegenerative diseases may have subtle

\footnotetext{
${ }^{1}$ Department of Biomedical Sciences, Neuroscience Research Institute, Seoul National University College of Medicine, Seoul, Korea; ${ }^{2}$ Department of Biomedical Laboratory Science, College of Health Science, Yonsei University, Wonju, Korea; ${ }^{3}$ Department of Neuroscience and Institute for Translational Neuroscience, University of Minnesota, Minneapolis, MN, USA and ${ }^{4}$ Department of Anatomy, School of Medicine, Konkuk University, Seoul, Korea Correspondence: Professor S-J Lee, Department of Biomedical Sciences, Neuroscience Research Institute, Seoul National University College of Medicine, Medical Science Building, Rm B101, 103 Daehak-ro, Jongro-gu, Seoul 03080, Korea.

E-mail: sjlee66@snu.ac.kr

Received 12 September 2016; revised 26 October 2016; accepted 15 November 2016
} 
morphological distinctions. However, the actual functional and structural heterogeneity of the protein aggregates in pathogenesis has not been determined. One of the biggest obstacles in this field of research is the difficulty in obtaining an abundance of in vivo samples from the brains of patients. Even if a miniscule amount of sample were available through autopsy, it would be unreliable and insufficient for studying the various structures of amyloid fibrils at the molecular level. In the case of synucleinopathies, which encompass several neurological diseases, the detection of multiple structures of different types of $\alpha$-synuclein fibrils may be of critical importance.

Several years ago, the advent of the protein misfolding cyclic amplification (PMCA) technique made it possible to amplify prion fibrils and detect minute amounts of prion from biological specimens. ${ }^{10}$ Here we developed a PMCA procedure to quantitatively and qualitatively multiply $\alpha$-synuclein fibrils and demonstrated the validation of this methodology. The present study displays the effectiveness of PMCA in the consistency of $\alpha$-synuclein fibril conformation and their replication into large quantities. These results further suggest that this revised version of the PMCA procedure may provide a strategy for examining the structural and functional heterogeneity of $\alpha$-synuclein aggregates.

\section{MATERIALS AND METHODS}

\section{Materials}

Anti- $\alpha$-synuclein monoclonal antibody was purchased from BD Biosciences (\#610787, San Diego, CA, USA). Isopropyl-1-thio- $\beta$-Dgalactopyranoside (IPTG), glycine, thioflavin $\mathrm{T}$ (ThT), proteinase $\mathrm{K}$ (PK) and protease inhibitor cocktail were purchased from SigmaAldrich (St Louis, MO, USA). Anion-exchange chromatography (HiTrap Q FF, \#17-5053-01) and Superdex-200 gel filtration chromatography (\#17-5175-01) columns were purchased from GE Healthcare (Fairfield, CT, USA).

\section{Purification of recombinant $\alpha$-synuclein}

Protein expression was induced with $0.1 \mathrm{~mm}$ IPTG for $3 \mathrm{~h}$ at $37^{\circ} \mathrm{C}$ when the absorbance of the E. coli strain BL21 (DE3) (RBC Korea, Seoul, Korea) culture reached 0.6 at $600 \mathrm{~nm}$. The cells were pelleted and resuspended in $20 \mathrm{~mm}$ sodium phosphate buffer $(\mathrm{pH} 7.4)$ for sonication and were subsequently boiled at $100^{\circ} \mathrm{C}$ for $20 \mathrm{~min}$, then centrifuged at $10000 \mathrm{~g}$ for $10 \mathrm{~min}$. The supernatant was subjected to anion-exchange chromatography and Superdex-200 gel filtration column chromatography for further purification. The purified $\alpha$-synuclein was dialyzed against distilled water and subsequently lyophilized.

For monomer preparation, lyophilized $\alpha$-synuclein was reconstituted in phosphate-buffered saline (PBS; \#CAP08-050, GenDEPOT, Katy, TX, USA), then subjected to ultrafiltration using a 100,000 molecular weight cut-off centrifugal device (Pall, New York, USA). For fibrillation, $\alpha$-synuclein $\left(200 \mu \mathrm{M}\right.$ in PBS) was incubated at $37^{\circ} \mathrm{C}$ for 19 days with constant shaking at 1,050 r.p.m. in a Thermomixer C (\#5382000015, Eppendorf, Hamburg, Germany). When used as seeds, the fibrils were sonicated for $1 \mathrm{~min}$ (Amplitude 30\%) before treated to fibrillation reaction.

\section{Mouse strains}

(THY1-SNCA A53T) F53Sud/JA53T +/ - mice were purchased from Jackson Laboratory (Bar Harbor, ME, USA). Human $\alpha$-Syn (A53T) transgenic line G2-3 was produced and maintained as described in Lee et al. ${ }^{11}$

\section{Intramuscular injection}

Intramuscular inoculation of preformed fibrils of $\alpha$-synuclein was performed as described in Sacino et al., ${ }^{12}$ with some modifications. The experimental protocol was approved by the Animal Care and Use Committee of the University of Minnesota (Protocol \#1602-33506A). Briefly, recombinant $\alpha$-synuclein and preformed fibril ( $\mathrm{pFF}$ ) preparations were injected into the biceps femoris muscles of 4 to 6-month-old transgenic mice over expressing the A53T mutant of human $\alpha$-Syn (line G2-3). ${ }^{11}$ The animals were anesthetized with isoflurane and injected into both biceps femoris (each biceps femoris received $5 \mu \mathrm{g}$ of pFF in $5 \mu \mathrm{l} \mathrm{PBS}$ ) through a 27 -gauge needle attached to a $10 \mu \mathrm{l}$ Hamilton syringe via polyethylene tubing $(0.38 \times 1.09 \mathrm{~mm})$. After injections, the animals were allowed to recover on a heating pad before being returned to their cages. The injected $\alpha$-synuclein transgenic mice developed overt motor dysfunction within 90 days post inoculation

\section{Preparation of brain tissues as exogenous seeds}

Brain and spinal cord tissues were homogenized with a tissue grinder (\#358039, Wheaton, Millville, NJ, USA) to $10 \%$ weight/volume (w/v) solution with homogenizing buffer ( $1 \%$ Triton X-100, $150 \mathrm{~mm} \mathrm{NaCl}$, Protease Inhibitor Cocktail). The protein concentration was determined by using a bicinchoninic acid assay (Pierce, Rockford, IL, USA).

\section{ThT binding assay}

Forty microliters of a $10 \mu \mathrm{M}$ recombinant $\alpha$-synuclein sample or $2.5 \mu \mathrm{M}$ PMCA products was added to $50 \mu \mathrm{l}$ of $10 \mu \mathrm{M}$ ThT solution in glycine- $\mathrm{NaOH}$ ( $\mathrm{pH} 8.5$ ). After $5 \mathrm{~min}$ of incubation, fluorescence was measured at $450 \mathrm{~nm}$ excitation and $490 \mathrm{~nm}$ emission settings.

\section{PMCA}

The equipment for PMCA, including a microplate horn (\#431MPX), sound enclosure (\#432MP) and thermoelectric chiller (\#4900), was purchased from Qsonica (Qsonica, Newtown, CT, USA). PMCA was performed using recombinant $\alpha$-synuclein monomers as substrates and recombinant $\alpha$-synuclein fibrils or tissue homogenates of transgenic as exogenous seeds. $\alpha$-Synuclein monomers were prepared to a final concentration of $20 \mu \mathrm{M}$ in conversion buffer ( $1 \%$ Triton X-100 and $150 \mathrm{~mm} \mathrm{NaCl}$ ), and $100 \mu \mathrm{l}$ of this sample transferred into siliconized PCR tubes. The samples for PMCA were subjected to cycles of $20 \mathrm{~s}$ sonication (Amplitude 50\%) and $29 \mathrm{~min} 40 \mathrm{~s}$ incubation at $37^{\circ} \mathrm{C}$ for a day or several days. Exogenous fibril seeds were added to final concentrations of $0.04 \%, 0.2 \%$ or $1 \%$.

\section{PK digestion}

Twenty micromolar $\alpha$-synuclein samples were incubated with final concentrations of 10 or $100 \mu \mathrm{g} \mathrm{ml} l^{-1}$ PK for $30 \mathrm{~min}$ to $2 \mathrm{~h}$ at $37^{\circ} \mathrm{C}$. Subsequently, sodium dodecyl sulfate (SDS)-PAGE buffer was added to the samples, which were then heated for $10 \mathrm{~min}$ at $95^{\circ} \mathrm{C}$.

\section{Immunoblotting}

Immunoblotting was performed as previously described. ${ }^{13}$ Protein samples (150 ng) were loaded onto 12\% SDS-PAGE gels for non-PK treated samples or $16 \%$ SDS-PAGE gels for PK-treated samples. 
Image detection was performed using an Amersham imager 600 (GE Healthcare Life Sciences, Marlborough, MA, USA), and Multi Gauge (v3.0) software (Fujifilm, Tokyo, Japan).

\section{Circular dichroism}

The samples were diluted in PBS at $15 \mu \mathrm{M}$ and analyzed using a Chirascan plus spectropolarimeter (Applied Photophysics, Randalls Rd, Leatherhead, UK).

\section{Transmission electron microscopy (TEM)}

Briefly, the samples were adsorbed onto 200 mesh carbon-coated copper grids, then subjected to negative staining with $2 \%$ uranyl acetate for $1 \mathrm{~min}$. The prepared grids were observed using a JEM1010 transmission electron microscope (JEOL, Akishima, Tokyo, Japan).

\section{Statistics}

All data in the figures are presented as the mean \pm s.e.m. $P$ values were measured by using unpaired two-tailed Student's t-tests in GraphPad InStat version 3.05 software (San Diego, CA, USA).

\section{RESULTS}

\section{Characterization of $\alpha$-synuclein fibrils in vitro}

The intrinsic structure of $\alpha$-synuclein is indefinable; however, in diseased states, this protein forms amyloid fibrils enriched with $\beta$-sheet conformations. ${ }^{14,15}$ To verify the structural properties of this protein at a molecular level, a ThT binding assay, circular dichroism spectroscopy, immunoblotting and Transmission electron microscopy were conducted to examine monomeric and fibril forms, respectively. ThT fluorescence was prominently more intense in the fibrilized $\alpha$-synuclein than in the monomers, thus demonstrating that $\beta$-sheet structures are abundant in fibril forms of $\alpha$-synuclein (Figure 1a). Circular dichroism spectroscopy confirmed an increase in the amount of $\beta$-sheet conformation in the fibrils than in the monomers (Figure 1b). Subsequent immunoblotting showed the visual representation of the SDS-stable aggregates (Figure 1c). Limited PK digestion resulted in a specific pattern of PK-resistant fragments, whereas the monomers were completely degraded (Figure 1d). The TEM images

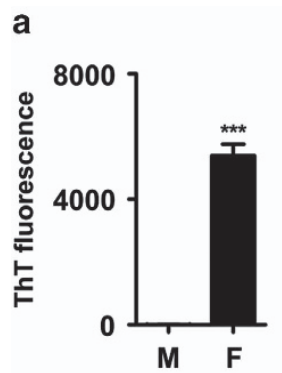

b

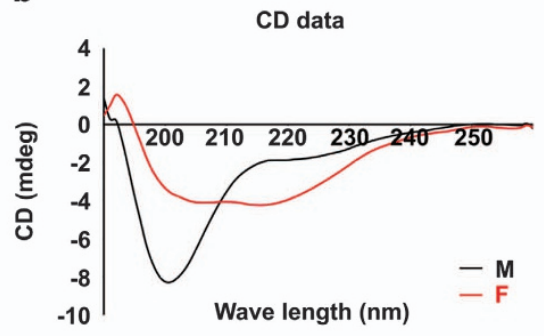

exhibited filamentous morphologies in a fibrilized form, whereas the monomers scarcely showed any discernable structures (Figure 1e). Together, the above data illustrate the characteristics of $\alpha$-synuclein in vitro, with clearly distinguishable features between its monomers and fibrils.

\section{The PMCA technique is readily applicable to the} amplification of $\alpha$-synuclein fibrils in vitro

To establish a procedure for cyclic amplification of $\alpha$-synuclein fibrils, we generated a modified PMCA protocol for $\alpha$-synuclein (Figure 2a). Over 48 cycles of PMCA, the fluorescence of ThT was markedly augmented when seed was added, whereas the seed-absent group scarcely exhibited any increase in fluorescence (Figure 2b). Western blotting confirmed that SDS-stable aggregates of $\alpha$-synuclein were formed during the PMCA cycles in the seed-added group (Figure 2c). When further observing the seed-added group via TEM, we visually identified multiple aggregates split into small fragments reflecting the repetitive sonication (Figure $2 \mathrm{~d}$ ). The amount of fibrils increased depending on the concentration of seeds, thus suggesting that the seeds are the main determinant of $\alpha$-synuclein fibrillation (Figure $2 \mathrm{e}$ and $\mathrm{f}$ ). Fibrils were able to be detected through PMCA at concentrations as low as $8 \mathrm{~nm}$. The PK digestion assay showed that the PMCA product with seed exhibited a PK digestion pattern similar to that of the seed fibril itself, whereas the monomers or PMCA products without seed did not result in PK-resistant bands (Figure 2g). These results suggest that the PMCA of $\alpha$-synuclein is highly sensitive and accurate in amplifying trace fibrils with conserved conformations.

PMCA products sustain the characteristics of the initial seed through multiple rounds

To assure the fidelity of perpetual replication of $\alpha$-synuclein fibrils, we performed consecutive rounds of PMCA, as illustrated in Figure 3a. The immunoblotting and ThT binding assays were conducted over three rounds, each comprising at least 48 cycles. The amounts of PMCA products
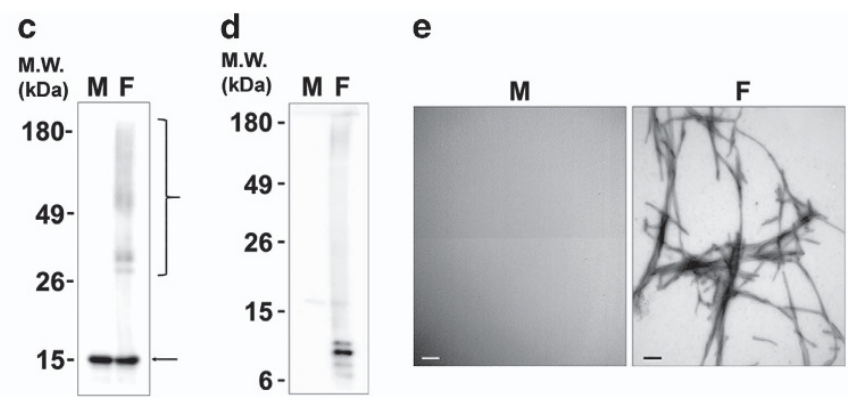

Figure 1 Characteristics of $\alpha$-synuclein monomers and fibrils. (a) ThT binding assay of recombinant $\alpha$-synuclein monomers and fibrils. $\mathrm{M}$ represents $\alpha$-synuclein monomers, and $\mathrm{F}$ represents $\alpha$-synuclein fibrils. Mean \pm s.e.m. of $n=4 ;{ }^{* * *} P<0.0001, t$-test. (b) CD spectra of monomers (black solid line) and fibrils (red solid line). CD spectra revealed that $\alpha$-synuclein monomers showed a typical random coil pattern, whereas fibrils exhibited $\beta$-sheet rich structures showing minimum absorbance at $218 \mathrm{~nm}$. (c) Immunoblotting of recombinant $\alpha$-synuclein monomers and fibrils. Black arrow, size of $\alpha$-synuclein monomer; Curly bracket, aggregates of $\alpha$-synuclein. (d) PK digestion patterns of monomers and fibrils. $20 \mathrm{~mm}$ samples were digested with $10 \mu \mathrm{g} \mathrm{ml}-1$ PK for $30 \mathrm{~min}$. PK-resistant fragments are shown in PK-treated fibrils, whereas monomers were completely degraded. (e) TEM images of recombinant $\alpha$-synuclein monomers (left) and fibrils (right). Fibrils showed a filamentous structure; Scale bars, $200 \mathrm{~nm}$. 

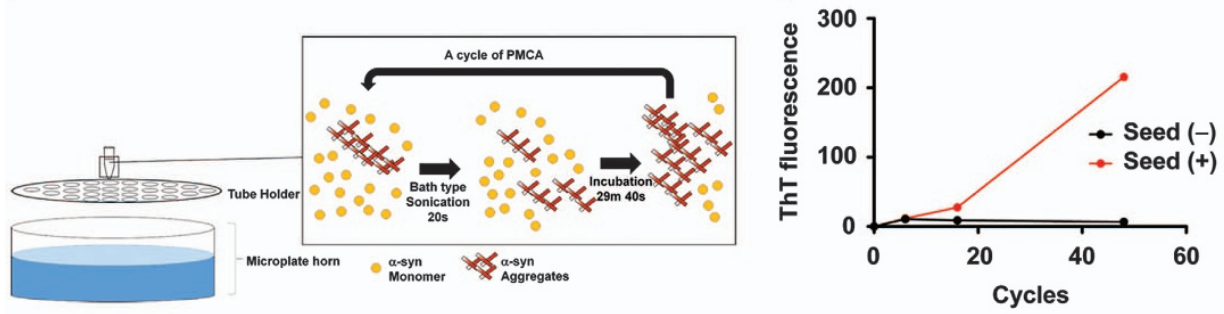

d

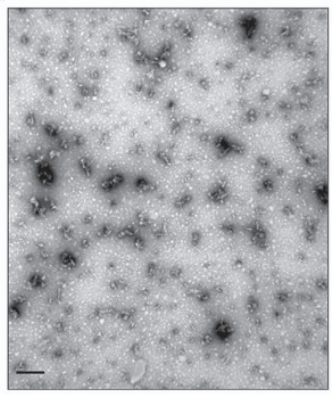

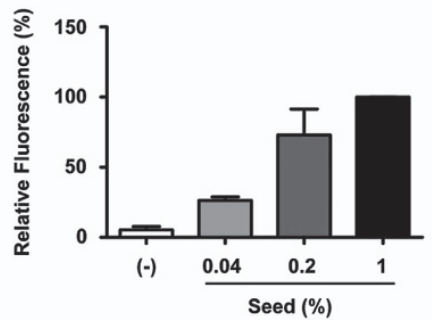

f

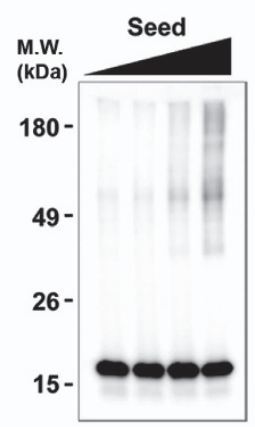

C M.W. $\quad \frac{0}{-+} \quad \frac{6}{-+} \quad \frac{16}{-+} \quad \underline{48} \begin{aligned} & \text { Cycles } \\ & \text { Seed }\end{aligned}$

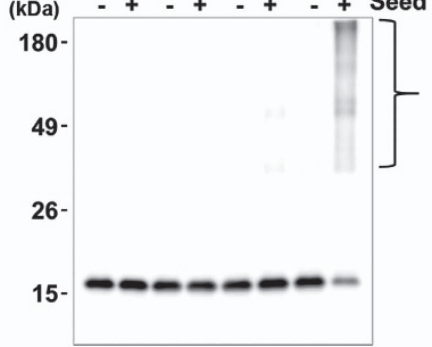

g
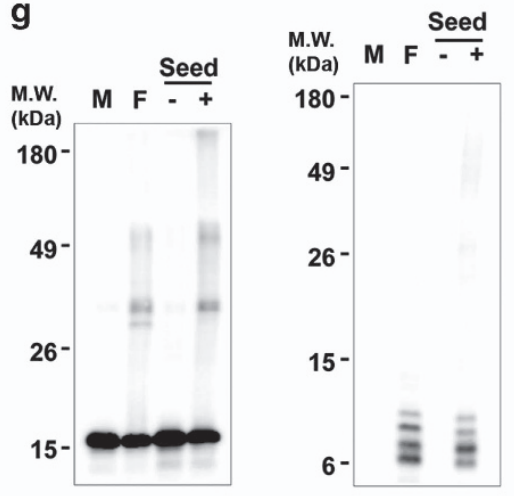

Figure 2 In vitro $\alpha$-synuclein fibrils are amplified using PMCA. (a) A schematic representation of the PMCA procedure. (b) ThT binding kinetics of recombinant $\alpha$-synuclein with or without sonicated fibril seeds after 48 cycles of PMCA. ThT fluorescence increased in the seed-added group, whereas the seed-absent group scarcely showed any increase in ThT fluorescence $(n=3)$. (c) Immunoblotting of PMCA products with indicated cycles of PMCA. Aggregated $\alpha$-synuclein was detectable after 48 cycles in samples with seeds. (d) TEM image of the seed-added group; scale bar, $200 \mathrm{~nm}$. (e) Relative ThT fluorescence was quantified in PMCA products after 48 cycles with different quantities of seeds. ThT fluorescence increased with increasing seed concentration $(n=3)$. (f) Immunoblotting of PMCA end products after 48 cycles with the same concentrations of seeds as in e. (g) Immunoblotting of PMCA product without (left) and with PK digestion (right). Similar PK-resistant fragments were detected in fibrils (F, seed itself) and the seed-added PMCA products, whereas nothing remained in monomers $(\mathrm{M})$ and seed-absent PMCA products.

increased with the number of cycles. Furthermore, the successive rounds of PMCA produced increasing amounts of aggregates (Figure $3 \mathrm{~b}$ ). The results of the ThT assay confirmed that the PMCA products maintained their state as fibrils and that a higher number of rounds results in higher fluorescence within a lower number of cycles (Figure 3c). To further analyze the structures of the amplified $\alpha$-synuclein fibrils, we performed a PK digestion, which revealed that the conformations of the PMCA end products were conserved after repeated rounds (Figure 3d).

\section{Conventional seeded aggregation is possible using the product of PMCA}

The feasibility of producing large quantities of fibrils was assessed through conventional seeded fibrillation using the PMCA products as seeds. The classical curve of nucleationdependent fibrillation was epitomized using the ThT binding assay in the absence of added seeds, whereas seeded aggregation with added PMCA products displayed a drastically reduced lag phase, reaching the fibril growth phase more rapidly (Figure 4a). The subsequent PK digestion verified that the conventional seeded aggregation produced fibrils that shared similar structures with the PMCA products (Figure 4b).
The TEM image of the seeded aggregates further confirmed the structure of the fibrils (Figure 4c). Together, these results indicate that products of PMCA can be indefinitely amplified through classical seeded aggregation.

\section{Cyclic amplification of in vivo fibrils}

Finally, we attempted to amplify $\alpha$-synuclein fibrils from tissue samples. Two different transgenic mouse models were utilized for in vivo experiments: Thy1-A53T expresses the A53T variant of human $\alpha$-synuclein under the Thyl promoter and mPrP-A53T expresses the same protein under a mouse PrP promoter. Thy1-A53T mice develop paralysis and die at the age of $9-11$ months. ${ }^{16}$ The brain that we used was obtained from 11-month-old animals that were killed after developing paralysis. For the mPrP-A53T mice, ${ }^{11}$ aggregation was induced through the intramuscular injection of in vitro-generated $\alpha$-synuclein fibrils. ${ }^{12}$ PMCA was performed using the tissue samples from the whole brain and the spinal cord of the Thyl-A53T and mPrP-A53T, respectively. Three rounds of PMCA were conducted for each mouse model with cycle numbers indicated in Figure $5 \mathrm{a}$ and $\mathrm{b}$. Aggregates were amplified from both tissue samples in a cycle numberdependent manner (Figure $5 \mathrm{a}$ and $\mathrm{b}$ ). PK digestion analysis 
b
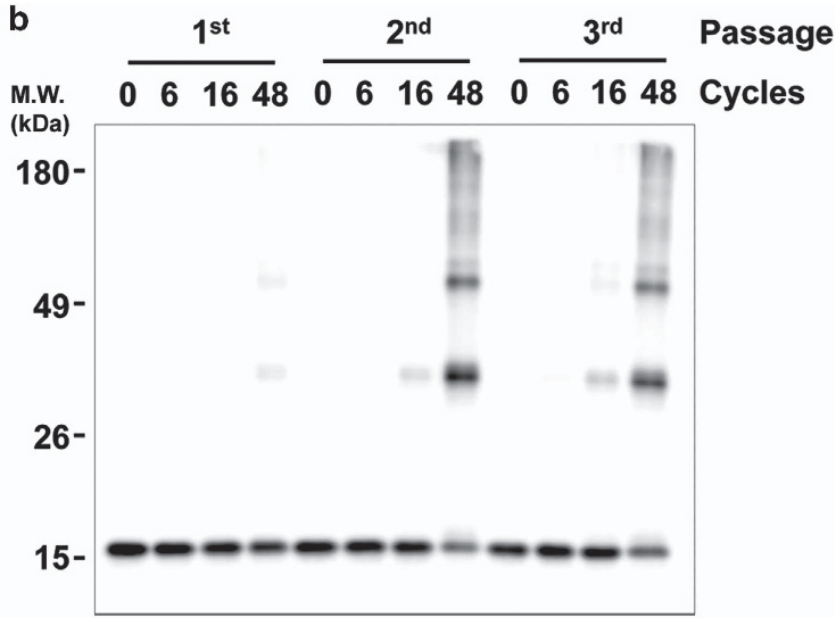

a

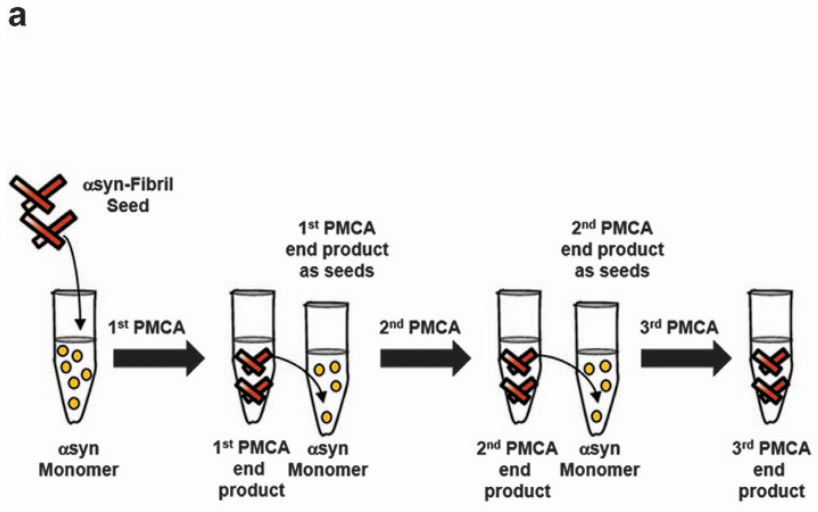

C

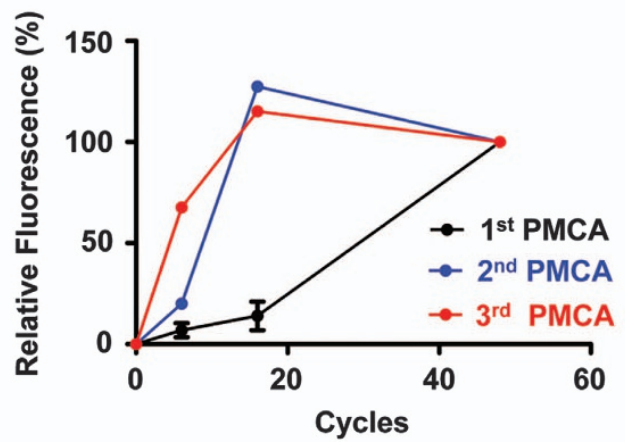

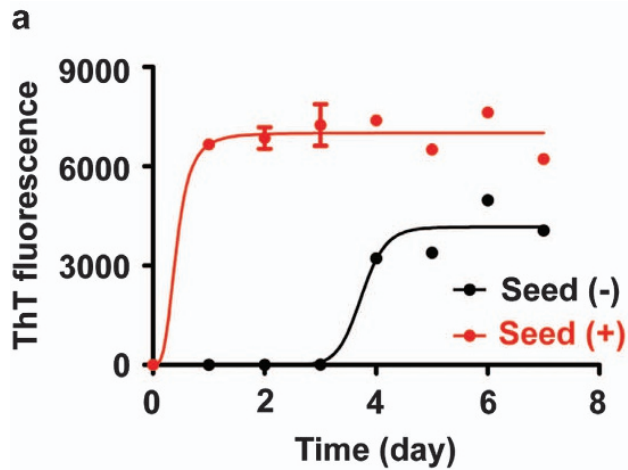

b

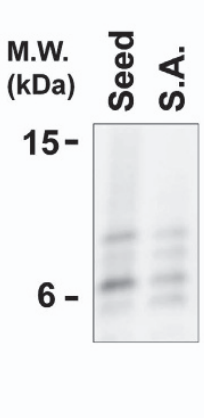

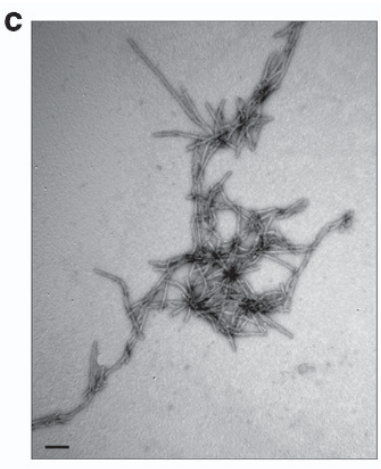

Figure 4 PMCA-amplified $\alpha$-synuclein aggregates have seeding activity in conventional fibrillation. (a) ThT binding kinetics of recombinant $\alpha$-synuclein with or without PMCA products as seeds $(n=3)$. (b) PK digestion patterns of seeded aggregates (S.A.) and PMCA products (Seed). PK-resistant fragments of the seeds were comparable to the seeded aggregates. (c) TEM images of seeded aggregates. The seeded aggregates showed filamentous structures; scale bar, $200 \mathrm{~nm}$.

exhibited different digestion patterns, thus indicating that the fibrils amplified from these mouse models were distinct conformers (Figure 5c). Both PMCA products were subjected to conventional seeded fibrillation. This procedure produced $\beta$-sheet-rich fibrils from both the PMCA products (Figure $5 \mathrm{~d}$ ), and each conventional seeded fibrillation generated fibrils that exhibited the same PK digestion pattern as the corresponding seed (the PMCA product) (Figure 5e). These results indicate that $\alpha$-synuclein fibrils can be amplified from the tissue samples with their fibril conformations conserved. 


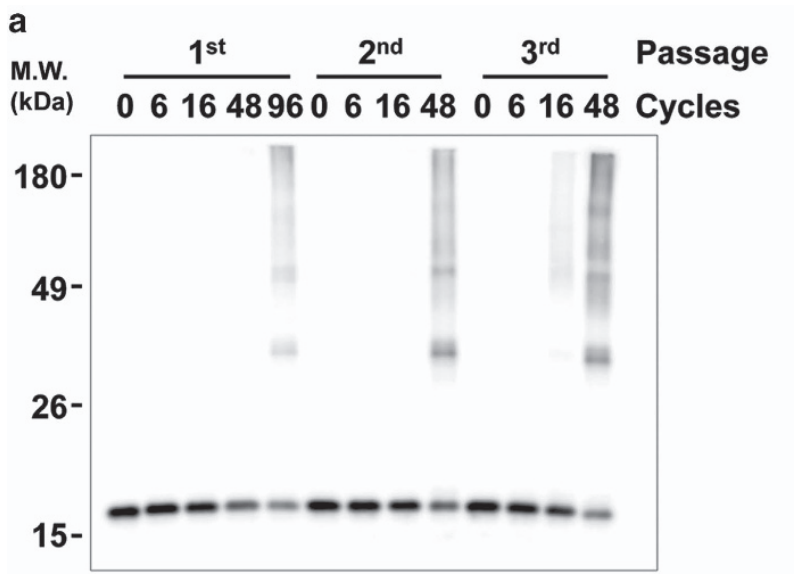

b

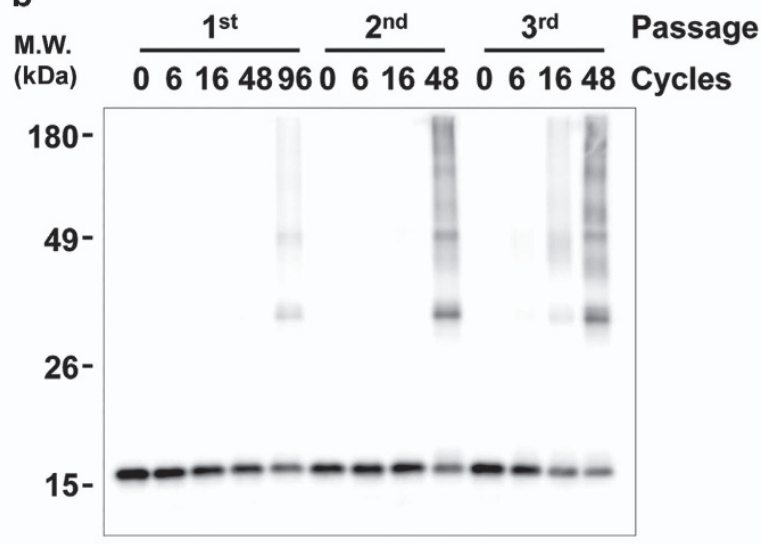

C

d
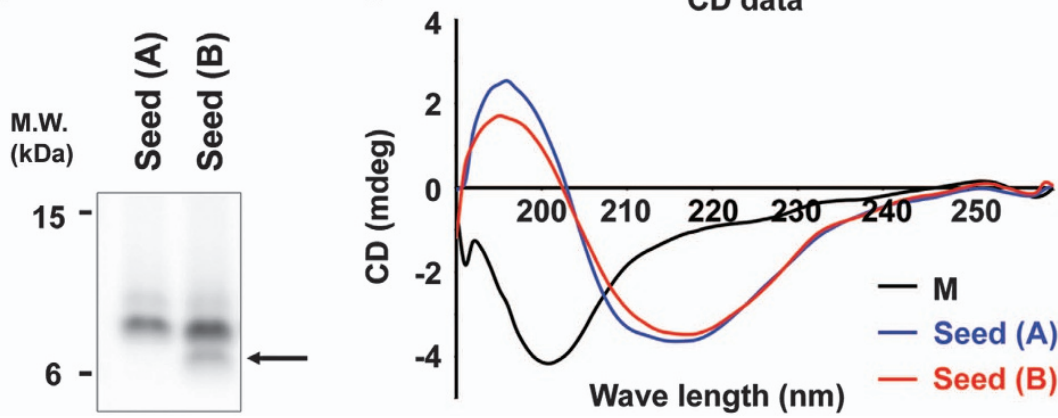

e

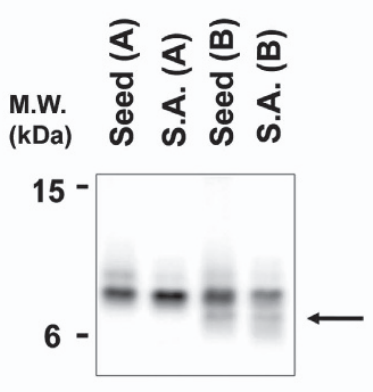

Figure 5 PMCA generates $\alpha$-synuclein aggregates from in vivo samples. (a) Immunoblotting of Thy1-A53T mice brain homogenate (seed A) induced sPMCA products with the indicated cycles. (b) Immunoblotting of mPrP-A53T spinal cord homogenates (seed B) induced sPMCA products with indicated cycles. (c) PK digestion patterns of PMCA products with different tissue seeds. Note the extra band (black arrow) on seed B-derived PMCA products. (d) CD spectra revealed seeded aggregates with different PMCA products and seeds possessing a $\beta$-sheet rich structure. (e) PK digestion patterns of seeded aggregates with different PMCA seeds. Seeded aggregates with different seeds exhibited similar PK resistant fragments with their respective seeds.

Table 1 Comparison of $\alpha$-synuclein PMCA methods

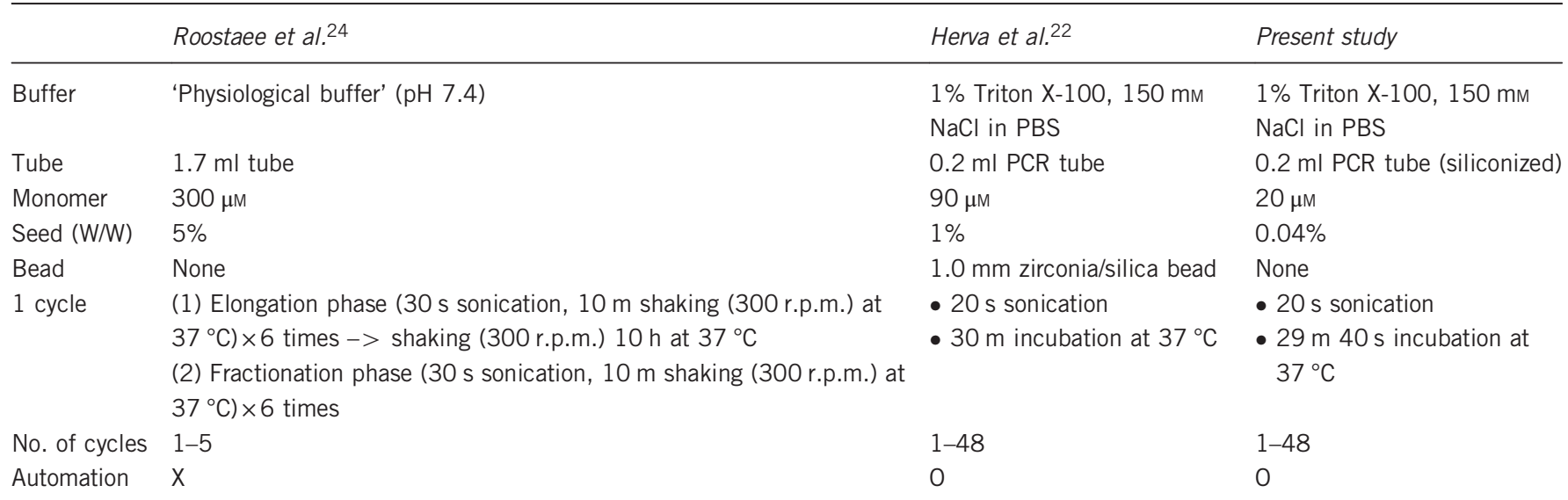

\section{DISCUSSION}

To date, research exploring various structures of fibrils has primarily been conducted on in vitro materials derived from synthetic peptides or recombinant proteins expressed in bacteria. In vivo-generated fibrils, however, have not been thoroughly investigated, owing to the scarcity of samples from patient brain tissues or animal models. In the present study, we generated and amplified $\alpha$-synuclein fibrils both in vitro and in vivo by implementing a PMCA technique. Experimental verification indicated that the PMCA products showed the same conformations as those of the seeds added to the reaction. Hence, we demonstrated that PMCA can be conducted 
through several rounds without the alteration of the original seed. These results suggest that a trace amount of in vivo fibrils can be amplified, and thus their specific structures can be determined.

In the present study, proteinase $\mathrm{K}$ digestion patterns were used to monitor structural diversities among fibrils. Although this method has some limitations in specifically identifying distinct fibril structures, PK digestion has nevertheless been widely and faithfully used to examine fibril conformers not only for prions but also for other amyloid fibrils, such as $\alpha$-synuclein. ${ }^{17,18}$ For higher accuracy, high-resolution techniques, such as solid-state nuclear magnetic resonance (ssNMR), may be used to determine fibril structures.

The PMCA technique was originally developed to amplify prions from tissues or body fluids. Although this technique has been used to amplify prions from various sources, it has also been applied to non-prion amyloid proteins, including $\mathrm{A} \beta,{ }^{19,20}$ tau $^{21}$ and $\alpha$-synuclein. ${ }^{22}$ In a previous study, Herva et al. ${ }^{22}$ have used in vitro-generated $\alpha$-synuclein fibrils as seeds in $\alpha$-synuclein PMCA (Table 1). This study successfully showed that $\alpha$-synuclein fibrils can be rapidly amplified from synthetic seeds and that the PMCA products 'infect' cells and consequently generate cytoplasmic aggregates. Although this study notably has demonstrated that PMCA can be applied to $\alpha$-synuclein, it has drawbacks in not addressing sensitivity and fidelity. The novelty of the present study is the amplification of in vivo-derived $\alpha$-synuclein fibrils and the demonstration of high sensitivity and fidelity. In the present study, we demonstrated that as little as $0.04 \%$ monomer can be amplified from trace seeds using the PMCA procedure. Moreover, we showed the amplification of two distinct $\alpha$-synuclein fibrils in two different transgenic mouse models and the production of PK digestion patterns unique to each model. The products from the initial PMCA can also be used as seeds in the next successive rounds of amplification and through several rounds, fibril conformations are faithfully replicated. These results indicate that $\alpha$-synuclein fibrils can be amplified from both in vitro and in vivo sources with high sensitivity and fidelity.

There are two notable aspects of the current study. Primarily, $\alpha$-synuclein PMCA made it possible to concretely determine the structures of in vivo fibrils. Recently, $A \beta$ fibrils have been isolated and amplified from two $\mathrm{AD}$ patients with distinct clinical manifestations. ${ }^{20}$ The determination of the structures of these fibrils through ssNMR has revealed that the fibrils were indeed distinguishable from each other in terms of conformations. On the basis of these observations, the authors have suggested that the different clinical features of the patients reflected distinct fibrils conformers, thus potentially representing two distinct 'strains'. To perform structural studies with such high resolution, large quantities, over several milligrams, of protein are required. Such quantities, however, are impossible to obtain from several rounds of PMCA. The products themselves should be further amplified through conventional seeded fibrillation. In the present study, we demonstrated the feasibility of attaining a large quantity of $\alpha$-synuclein fibrils through the conventional seeded fibrillation of $\alpha$-synuclein PMCA product without changing the fibril structure.

Second, the study demonstrated the clinical potential of the amplified $\alpha$-synuclein fibrils from biological samples, which might be strategically used for diagnosing synucleinopathies. The aggregation of $\alpha$-synuclein is associated with PD, DLB, MSA and $\mathrm{AD}^{3}{ }^{3}$ It is difficult to precisely pinpoint each synucleinopathy in diagnostics solely on the basis of the symptoms observed. In prion diseases, PMCA exemplified the prospective diagnostic detection of pathogenic prions from body fluids. ${ }^{23}$ Consequently, we expect that a similar approach using the PMCA technique can be adapted to synucleinopathies, thereby bringing the field one step closer to developing differential diagnostic tools.

\section{CONFLICT OF INTEREST}

The authors declare no conflict of interest.

\section{ACKNOWLEDGEMENTS}

We would like to thank Somin Lim for assistance in manuscript preparation. This work was financially supported by a National Research Foundation (NRF) grant funded through the Korean Government (MEST) (nos 2015R1A2A10052540 and 2015R1A2A15053661), the Korea Health Technology R\&D Project, Ministry of Health \& Welfare, Republic of Korea (HI14C0093) and Seoul National University Hospital.

1 Ross CA, Poirier MA. Protein aggregation and neurodegenerative disease. Nat Med 2004; 10(Suppl): S10-S17.

2 Hardy J. A hundred years of Alzheimer's disease research. Neuron 2006; 52: 3-13.

3 Lashuel HA, Overk CR, Oueslati A, Masliah E. The many faces of alpha-synuclein: from structure and toxicity to therapeutic target. Nat Rev Neurosci 2013; 14: 38-48.

4 Balch WE, Morimoto RI, Dillin A, Kelly JW. Adapting proteostasis for disease intervention. Science 2008; 319: 916-919.

5 Chiti F, Dobson CM. Protein misfolding, functional amyloid, and human disease. Annu Rev Biochem 2006; 75: 333-366.

6 Jarrett JT, Lansbury PT Jr. Seeding 'one-dimensional crystallization' of amyloid: a pathogenic mechanism in Alzheimer's disease and scrapie? Cell 1993; 73: 1055-1058.

7 Jucker M, Walker LC. Self-propagation of pathogenic protein aggregates in neurodegenerative diseases. Nature 2013; 501: 45-51.

8 Sanders DW, Kaufman SK, Holmes BB, Diamond MI. Prions and protein assemblies that convey biological information in health and disease. Neuron 2016; 89: 433-448.

9 Petkova AT, Leapman RD, Guo Z, Yau WM, Mattson MP, Tycko R. Self-propagating, molecular-level polymorphism in Alzheimer's betaamyloid fibrils. Science 2005; 307: 262-265.

10 Saborio GP, Permanne B, Soto C. Sensitive detection of pathological prion protein by cyclic amplification of protein misfolding. Nature 2001; 411 : 810-813.

11 Lee MK, Stirling W, Xu Y, Xu X, Qui D, Mandir AS et al. Human alpha-synuclein-harboring familial Parkinson's disease-linked Ala-53 -> Thr mutation causes neurodegenerative disease with alpha-synuclein aggregation in transgenic mice. Proc Natl Acad Sci USA 2002; 99: 8968-8973.

12 Sacino AN, Brooks M, Thomas MA, McKinney AB, Lee S, Regenhardt RW et al. Intramuscular injection of alpha-synuclein induces CNS alpha-synuclein pathology and a rapid-onset motor phenotype in transgenic mice. Proc Natl Acad Sci USA 2014; 111: 10732-10737. 
13 Lee HJ, Shin SY, Choi C, Lee YH, Lee SJ. Formation and removal of alpha-synuclein aggregates in cells exposed to mitochondrial inhibitors. J Biol Chem 2002; 277: 5411-5417.

14 Conway KA, Harper JD, Lansbury PT Jr. Fibrils formed in vitro from alphasynuclein and two mutant forms linked to Parkinson's disease are typical amyloid. Biochemistry 2000; 39: 2552-2563.

15 Weinreb PH, Zhen W, Poon AW, Conway KA, Lansbury PT Jr. NACP, a protein implicated in Alzheimer's disease and learning, is natively unfolded. Biochemistry 1996; 35: 13709-13715.

16 Chandra S, Gallardo G, Fernandez-Chacon R, Schluter OM, Sudhof TC. Alpha-synuclein cooperates with CSPalpha in preventing neurodegeneration. Cell 2005; 123: 383-396.

17 Collinge J, Sidle KC, Meads J, Ironside J, Hill AF. Molecular analysis of prion strain variation and the aetiology of 'new variant' CJD. Nature 1996; 383: 685-690

18 Bousset L, Pieri L, Ruiz-Arlandis G, Gath J, Jensen PH, Habenstein B et al. Structural and functional characterization of two alpha-synuclein strains. Nat Commun 2013; 4: 2575.

19 Paravastu AK, Qahwash I, Leapman RD, Meredith SC, Tycko R. Seeded growth of beta-amyloid fibrils from Alzheimer's brain-derived fibrils produces a distinct fibril structure. Proc Natl Acad Sci USA 2009; 106: 7443-7448.

20 Lu JX, Qiang W, Yau WM, Schwieters CD, Meredith SC, Tycko R. Molecular structure of beta-amyloid fibrils in Alzheimer's disease brain tissue. Cell 2013; 154: 1257-1268.
21 Meyer V, Dinkel PD, Rickman Hager E, Margittai M. Amplification of Tau fibrils from minute quantities of seeds. Biochemistry 2014; 53 : 5804-5809.

22 Herva ME, Zibaee S, Fraser G, Barker RA, Goedert M, Spillantini MG. Anti-amyloid compounds inhibit alpha-synuclein aggregation induced by protein misfolding cyclic amplification (PMCA). J Biol Chem 2014; 289: 11897-11905.

23 Chen B, Morales R, Barria MA, Soto C. Estimating prion concentration in fluids and tissues by quantitative PMCA. Nat Methods 2010; 7: 519-520.

24 Roostaee A, Beaudoin S, Staskevicius A, Roucou X. Aggregation and neurotoxicity of recombinant alpha-synuclein aggregates initiated by dimerization. Mol Neurodegener 2013; 8: 5.

cc)(1)(2) This work is licensed under a Creative Commons Attribution-NonCommercial-ShareAlike $\quad \mathbf{4 . 0}$ International License. The images or other third party material in this article are included in the article's Creative Commons license, unless indicated otherwise in the credit line; if the material is not included under the Creative Commons license, users will need to obtain permission from the license holder to reproduce the material. To view a copy of this license, visit http://creativecommons.org/licenses/by-nc-sa/4.0/ 